Musées, Patrimoine et Culture scientifiques et techniques

$116 \mid 2008$

mars - avril 2008

\title{
Le nouveau musée des Monuments français
}

\section{Odile Welfelé}

\section{OpenEdition \\ Journals}

Édition électronique

URL : http://journals.openedition.org/ocim/371

DOI : 10.4000/ocim.371

ISSN : 2108-646X

Éditeur

OCIM

Édition imprimée

Date de publication : 1 mars 2008

Pagination : 21-27

ISSN : 0994-1908

Référence électronique

Odile Welfelé, « Le nouveau musée des Monuments français », La Lettre de l'OCIM [En ligne], 116 | 2008, mis en ligne le 01 janvier 2011, consulté le 10 décembre 2020. URL : http://journals.openedition.org/ ocim/371 ; DOI : https://doi.org/10.4000/ocim.371 
déménagement à Bercy, la Cinémathèque et le musée du Cinéma, le musée des Monuments français avait bien connu des alertes depuis la toute fin des années 1970 jusqu'au début des années 2000. Sa réouverture n'allait pas de soi.

La création de la Cité de l'Architecture et du Patrimoine imposait au musée de réfléchir à son nouveau destin : musée patrimonial ou musée d'architecture ? La nature même des collections de moulages et des copies de peintures, remontant pour les plus anciennes au XIX ${ }^{e}$ siècle, faisait pencher la balance en faveur d'un musée patrimonial. La création d'une nouvelle galerie, la galerie de l'Architecture moderne et contemporaine, semblait indiquer une volonté de tendre vers le musée d'architecture.

Que le musée ait aussi une vocation architecturale remonte pourtant à ses origines quand Anatole de Baudot (1834-1915) y crée la chaire d'histoire de l'Architecture et fait réaliser des maquettes didactiques en plâtre. Quelques décennies plus tard, Philippe Chapu, directeur de 1979 à 1992, en avait fait une de ses préoccupations sans arriver à véritablement la mettre en œuvre, faute de crédits et sans doute de soutiens institutionnels. Il réussit toutefois à arrêter le projet d'installation de l'école de Danse de Maurice Béjart qui aurait pris une partie des volumes du musée et qui suscita une mobilisation importante de la presse et de différents soutiens. Guy Cogeval, directeur de 1992 à 1998, participe au premier projet de création d'un Centre national du Patrimoine qui entraîne en 1995 la fermeture du musée pour procéder aux travaux nécessaires. Ce projet devient en 1997 celui d'une Cité de l'Architecture et du Patrimoine, soutenu par le ministère de la Culture et sa nouvelle direction de l'Architecture et du Patrimoine.

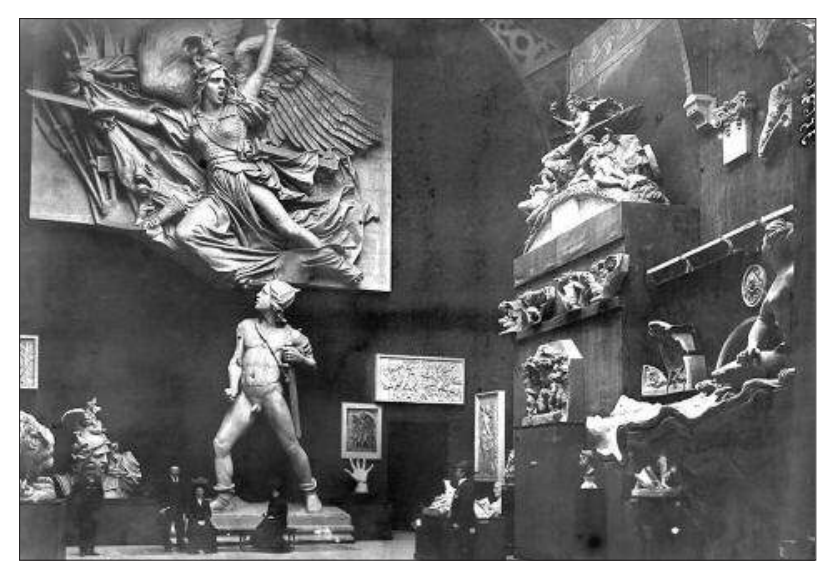

Le musée des Monuments français avant la construction de l'aile de 1937 (c) Ministère de la Culture, médiathèque de l'Architecture et du Patrimoine
Ces projets successifs, le manque de moyens financiers, la désaffection du public, la fermeture et l'incendie de 1995, ont alimenté les critiques sur le maintien des collections de moulages et de copies de peintures murales sur le site de Chaillot. L'intérêt des collections, parfois qualifiées de copies poussiéreuses, n'apparaissait plus.

Plus tard, une fois le projet de réutilisation des lieux stabilisé, il a fallu revoir, en quelques courtes années, l'ensemble du bâtiment destiné à accueillir d'autres structures que le musée: IFA, CEDHEC, ainsi que des ateliers pédagogiques, des espaces d'expositions temporaires, des lieux d'accueil pour les partenaires, une librairie et un restaurant, le tout dans une vocation architecturale contemporaine fortement affirmée. Le musée des Monuments français devait-il prétendre devenir un musée d'architecture ? La nouvelle muséographie saurait-elle atteindre le public qui l'avait déserté ?

On peut distinguer trois grandes orientations de la rénovation du musée : la simplification et l'harmonisation des présentations, la présence plus forte de l'architecture, la modernisation et l'enrichissement des dispositifs destinés au public. Le patrimoine devait s'allier au contemporain, non seulement dans ses collections mais aussi dans les outils de présentation.

La simplification a d'abord consisté à limiter, de manière parfois drastique, la présentation en salle du nombre d'œuvres des anciennes collections de moulages et de peintures. Il fallait sortir des anciennes propositions, à tendance pédagogique sinon encyclopédique, pour offrir un parcours facile à appréhender grâce à des œuvres judicieusement choisies. Le musée devait également se plier aux nouveaux impératifs de visites et de sécurité du public ainsi qu'aux prévisions d'utilisation des salles

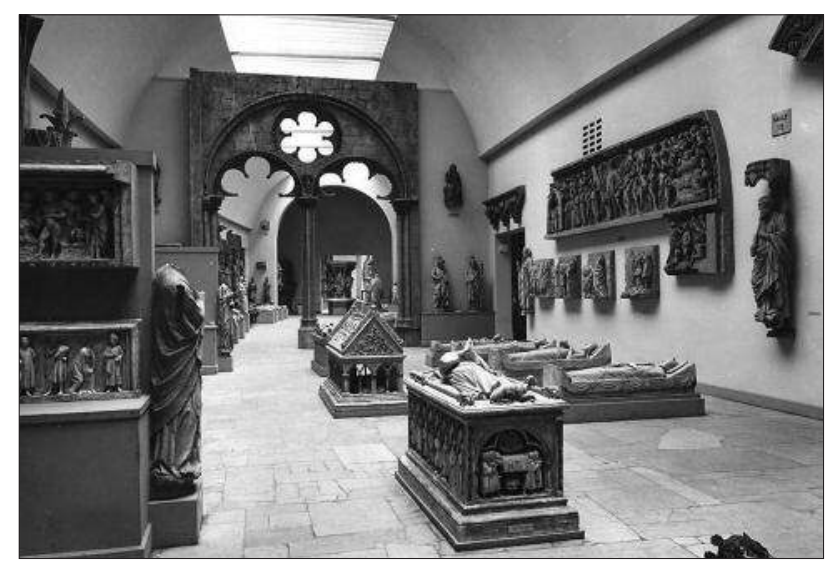

Une salle du musée vers 1970 ( ) CAPA/musée des Monuments français 
pour accueillir des évènements dont l'apport financier est à présent indispensable à la majorité des structures muséales. La création de la bibliothèque et de la nouvelle galerie de l'Architecture moderne et contemporaine ont entraîné une redistribution des espaces. Pour autant, il fallait apporter à ces collections une nouvelle dynamique et faciliter leur appréhension par un public peu au fait de ces techniques et de leurs objectifs.

\section{Une muséographie d'une spectaculaire simplicité pour les galeries des Moulages et des Peintures et vitraux}

La galerie des Moulages, au rez-de-chaussée, présente 350 moulages en plâtre à l'échelle 1 et 60 maquettes d'architecture et de charpentes. Les choix à faire ont été dictés par la nature des moulages et par la rénovation des espaces. Un des objectifs essentiels étant de faire entrer le musée dans le projet de la Cité de l'Architecture et du Patrimoine, les œuvres à garder prioritairement étaient les grands portails romans restés en place, les éléments monumentaux du Gothique et de la Renaissance. Le parcours reste de fait chronologique. Il est même régional dans les salles romanes, notion sans doute périmée mais qui ne pouvait être modifiée au regard du poids respectif de la taille des moulages et de l'espace disponible.

Les collections de la galerie, la plus ancienne et la plus importante des trois galeries, viennent essentiellement de celles du musée de Sculpture comparée ouvert en 1882 dans le tout nouveau Palais du Trocadéro. Pour 2007, de nombreuses œuvres ont dû être retirées des galeries. D'une part, l'accrochage au mur a été allégé, d'autre part la place disponible a été réduite, le niveau supérieur ayant été dévolu à la nouvelle galerie de l'Architecture contemporaine. La collection n'est plus présentée que dans les deux galeries du rez-de-chaussée, les galeries Davioud et Carlu. La galerie Carlu a été construite lors du remaniement du bâtiment entre 1935 et 1937, le long de la galerie Davioud, côté Seine, épousant la courbe de celle-ci. Pour présenter une quantité plus importante d'œuvres, des épis perpendiculaires aux fenêtres avaient été créés. Ils ont été supprimés et les moulages s'installent sur les murs de part et d'autre de la galerie, laissant une libre circulation centrale. La galerie Carlu se déroule ainsi sans rupture visuelle mais aussi sans réelle différenciation des périodes présentées et avec un nombre d'œuvres réduit.
La galerie Davioud, héritage de l'ancien Palais du Trocadéro construit en 1878, a fait l'objet d'une restauration tendant à restituer son état d'origine. Elle comporte un seul niveau de présentation des moulages, surplombé sur toute sa longueur de verrières, soutenues par des fermes métalliques ouvragées, qui apportent un éclairage zénithal. La galerie est composée de salles successives, qui permettent une scansion des périodes ou des thématiques. La nouvelle muséographie de 2007 propose un retour à la couleur rouge, généralement appelé « pompéien », des murs de la galerie Davioud et rend visibles les fermes métalliques qui avaient été masquées par du plâtre en 1937 pour harmoniser cette ancienne galerie avec la nouvelle galerie Carlu.

L'escalier construit par Jean-François Bodin, architecte responsable de la rénovation du bâtiment et de la nouvelle scénographie du musée, au milieu de la galerie et qui mène aux niveaux inférieurs et supérieurs, permet de marquer la césure du parcours chronologique proposé au public. La galerie supérieure de Carlu était précédemment dévolue aux

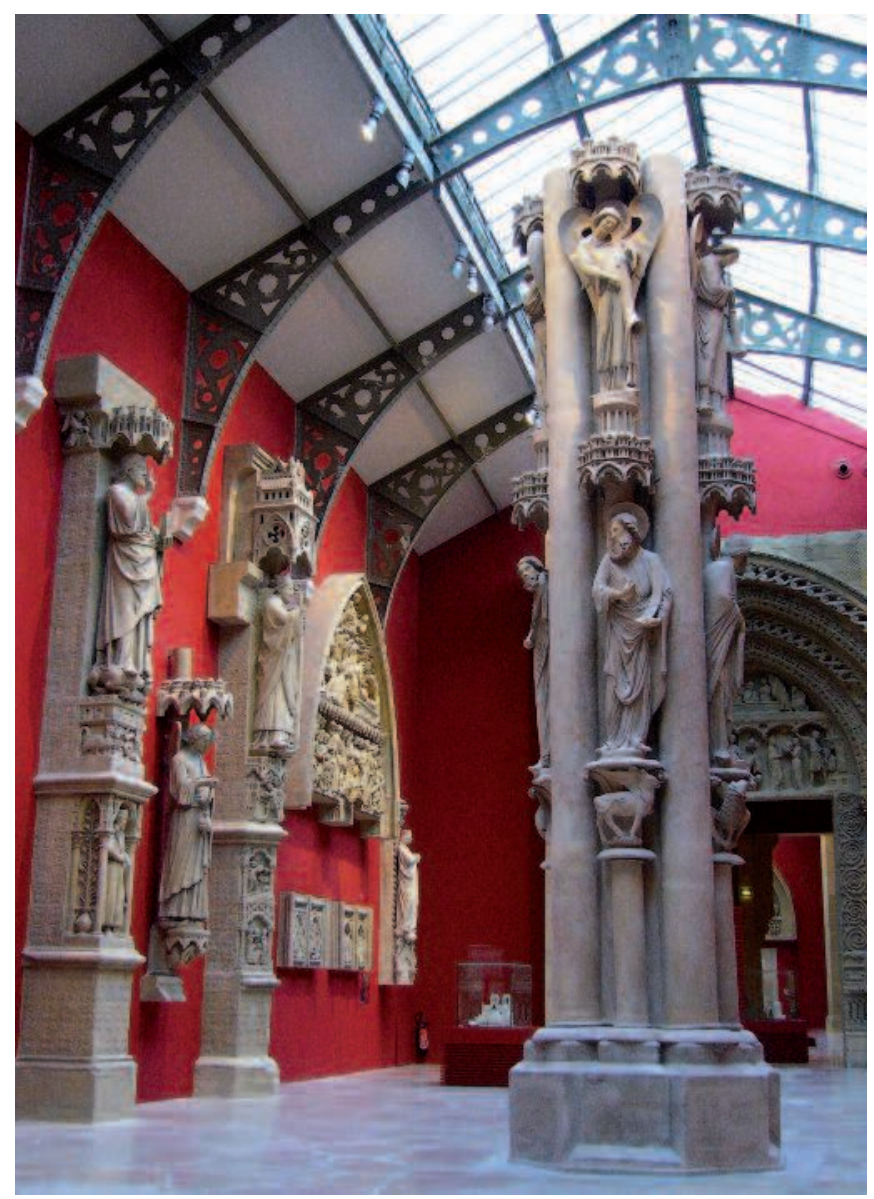

La galerie des Moulages : salle consacrée à la plénitude du Gothique avec le pilier des Anges de Strasbourg et la statuaire de la cathédrale d'Amiens. (c) CAPA/musée des Monuments français 
moulages des $\mathrm{XVII}^{e}, \mathrm{XVIII}^{e}$ et XIX ${ }^{e}$ siècles, présentant notamment la grandiose Marseillaise de Rude dont l'original est sur l'Arc de Triomphe de Paris. Son attribution aux collections de la nouvelle galerie d'Architecture moderne et contemporaine a contribué à réduire de manière drastique la présentation des œuvres des XVII ${ }^{e}$ et XVIII ${ }^{e}$ siècles et à la non-présentation d'œuvres du XIXe siècle. Le parcours chronologique s'arrête donc face à l'escalier avec la fontaine de Neptune de Nancy et la maquette d'opéra conçue par l'architecte Muly entre 1781 et 1785 (dépôt de la Bibliothèque nationale de France). Les maquettes en plâtre, de dimensions souvent imposantes, rythment le parcours en facilitant une dispersion du regard. Leur présence rappelle la place donnée à l'enseignement de l'architecture.

Renoncer à des périodes ne pouvait suffire. La présentation des collections devait être allégée. Tous les très petits moulages des époques médiévales et Renaissance ont, pour la grande majorité, été laissés en réserves. Les gisants et tombeaux, à l'exception notable du tombeau de Jean de Berry, et qui étaient placés au centre des espaces de circulation, n'ont pas non plus été replacés. La notion d'œuvres emblématiques a surpassé le concept encyclopédique.

La galerie des Peintures et des vitraux, sur deux niveaux, présente 80 copies de peintures murales romanes, gothiques et de la Renaissance à l'échelle 1 ainsi que 5 copies de vitraux. Dans la galerie des Moulages, deux copies de vitraux de la cathédrale de Chartres, et la copie de la peinture de la chapelle de

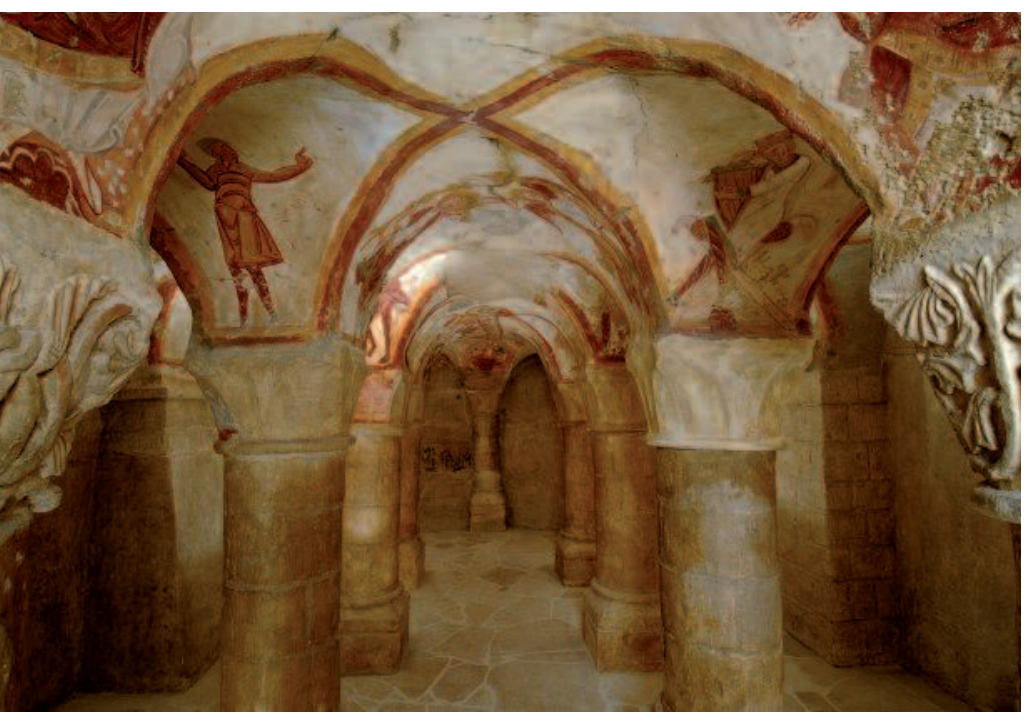

La galerie des Peintures : reconstitution, réalisée en 1941, de la crypte, datée de la première moitié du XIle siècle,

de l'église de Tavant (Indre-et-Loire) (c) CAPA/musée des Monuments français/B. Lomont
Berzé-la-Ville (Saône-et-Loire) ouvrent le dialogue avec la galerie des Peintures et vitraux. Toutes les copies de peintures ont fait l'objet d'une campagne totale de restauration, facilitée par le déménagement qui obligeait au déplacement des œuvres.

Les peintures en volume ont été ôtées de leur support et les chapelles en volume découpées. Le dos des lés a été entièrement dépouillé de sa colle végétale qui attirait des parasites. Les peintures ont ensuite été remises en place et restaurées après repose. La voûte de Saint-Savin-sur-Gartempe, déjà évoquée, la coupole de la cathédrale Saint-Étienne de Cahors, la crypte de l'église Saint-Nicolas de Tavant (Indre-et-Loire), la voûte de la chapelle du château de Pimpéan (Maine-et-Loire), et bien d'autres, offrent de nouveau leurs volumes exceptionnels et cette extraordinaire sensation de dépaysement, si propre à ce musée.

Quant aux peintures planes qui étaient précédemment présentées dans un cadre et donnaient l'illusion d'une peinture au chevalet, elles sont à présent installées au nu du mur. Elles peuvent en être désolidarisées pour permettre des prêts extérieurs. Le placement à hauteur d'œil des copies de vitraux permet d'en avoir une vision confortable ce qui n'est pas possible dans les édifices originaux.

\section{Des dispositifs de compréhension des œuvres nouveaux et interactifs}

Les cartels d'œuvre ont fait l'objet de recherches multiples, tant historiques que graphiques. Les anciens cartels étaient sommaires, présentant le nom de l'œuvre (cathédrale Notre-Dame), son implantation géographique (Amiens) et une datation simplifiée (XIII ${ }^{e}$ siècle). Ils tendaient à donner la primauté à l'édifice original en effaçant le moulage.

Tous les cartels d'œuvre portent à présent une petite carte de France qui indique la région où se situe l'édifice original. L'information très détaillée sur l'édifice situe précisément l'implantation de l'œuvre dont nous avons le moulage (par exemple : « trumeau $d u$ portail central de la façade occidentale »). Le nom du mouleur et la date d'entrée dans le musée, ainsi que le numéro d'inventaire et la date de classement de l'édifice, complètent les informations muséographiques sur l'œuvre.

Un grand nombre de cartels présente des éléments de compréhension de l'œuvre, de son système narratif ou parfois des conditions dans lesquelles elle a été moulée. Ces cartels détaillés comportent également une photographie de l'édifice qui permet de replacer le moulage dans le contexte d'une façade ou d'un 


\section{La Cité de l'Architecture et du Pairimoine en bref}

La Cité de l'Architecture et du Patrimoine est un centre de diffusion de la connaissance de la qualité de l'architecture, de la valorisation du patrimoine et de la préservation de l'environnement urbain. Ce vaste chantier - établissement public industriel et commercial, institué par décret du 9 juillet 2004, sous la tutelle du ministère de la Culture et de la Communication (direction de l'Architecture et du Patrimoine) - a été initié de 1999 à 2003 par Jean-Louis Cohen, architecte et historien. La scénographie du bâtiment et des galeries du musée est due à Jean-François Bodin, architecte.

La Cité dispose sur $23000 \mathrm{~m}^{2}$ de trois départements pour assurer les missions qui lui ont été confiées :

- l'institut français d'Architecture pour la diffusion et la promotion de la création architecturale contemporaine,

- le musée des Monuments français $\left(8000 \mathrm{~m}^{2}\right)$ pour la valorisation et la connaissance du patrimoine architectural français,

- l'École de Chaillot pour la formation des professionnels (architectes-urbanistes de l'État, architectes spécialisés dans la conservation et la restauration architecturale, urbaine et paysagère) et l'initiation du public (cours publics).

Une bibliothèque d'architecture et le centre d'archives d'architecture du XX ${ }^{e}$ siècle ainsi qu'un service des publics complètent ce dispositif de mise à disposition d'outils de connaissance et de culture de l'architecture et du patrimoine.

ensemble. On rejoint ainsi le souhait de Viollet-leDuc d'associer moulages et iconographie. Ces cartels sont de grande taille ce que permet la taille des œuvres. Le moulage acquiert ainsi sa place d'œuvre à part entière, reliée à la fois à l'édifice dont il est issu et à la constitution des collections.

Les fiches de lecture disponibles dans les salles qui proposent des informations plus poussées sur certaines œuvres ont fait l'objet d'une réflexion particulière. De taille importante, en couleurs et sur un support plastifié agréable au toucher, elles comportent 7000 signes environ et une iconographie riche. Depuis l'ouverture, on a pu constater que le public porte un grand intérêt aux cartels comme aux fiches.

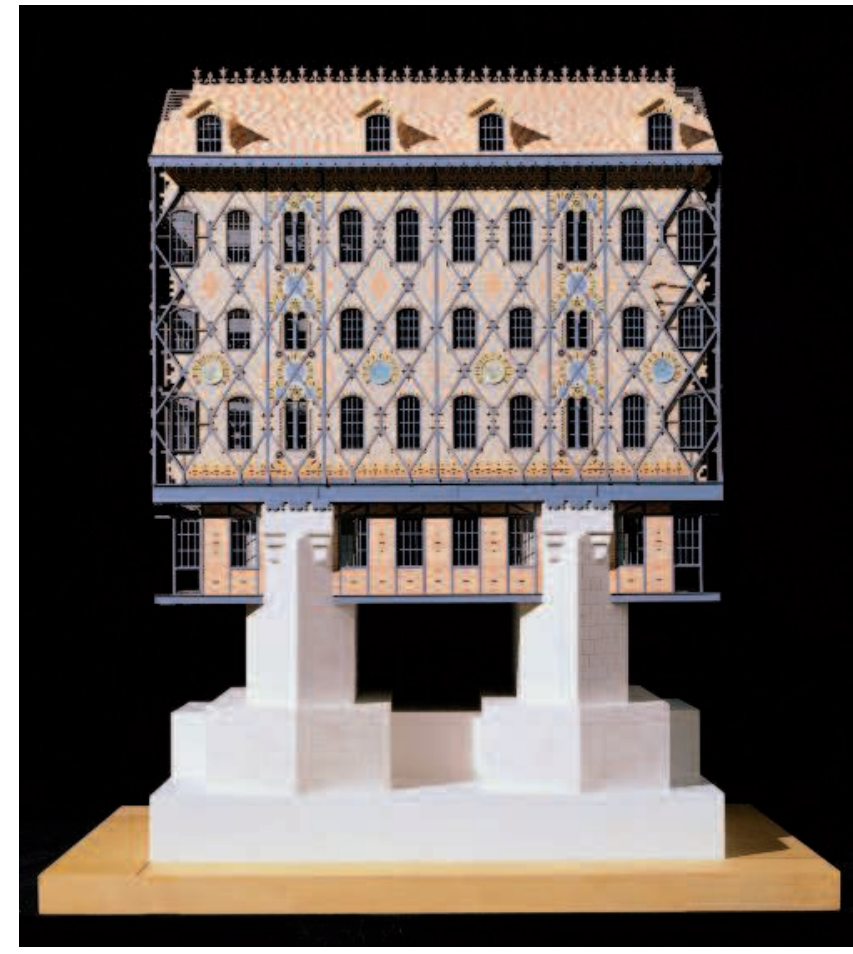

Maquette du bâtiment de la chocolaterie Meunier à Noisel (Jules Saulnier, architecte, 1871-1872) ( $)$ CAPA/G. Bergeret

Ces dernières ont même un tel succès qu'elles disparaissent en dépit de leur taille... Un projet d'édition de ces fiches pour la vente est à l'étude.

Les dispositifs multimédia interactifs présents parmi les moulages connaissent également un grand succès. Leur approche est aisée : les écrans de grand format proposent une image de très grande définition. Une image mobile est toujours présente sur l'écran, ce qui attire l'oeil. Trackball et poussoir permettent une circulation facile.

Certaines bornes proposent des photographies qui peuvent être agrandies fortement ainsi que des vues panoramiques immersives qui permettent de voir les détails les plus fins et de tourner à $360^{\circ}$ à partir d'un point de vue. Le public peut approcher au plus près les sculptures des façades de Chartres, se promener dans les tribunes de Conques et plonger son regard dans la nef de Strasbourg depuis le triforium - dont l'accès est interdit au public en raison de sa dangerosité - ou voir l'abbaye d'Aulnay-de-Saintonge dans son environnement champêtre. D'autres bornes sont didactiques et dédiées à des sujets thématiques : l'histoire du musée, les techniques contemporaines de moulages et de restauration, l'abbaye du 

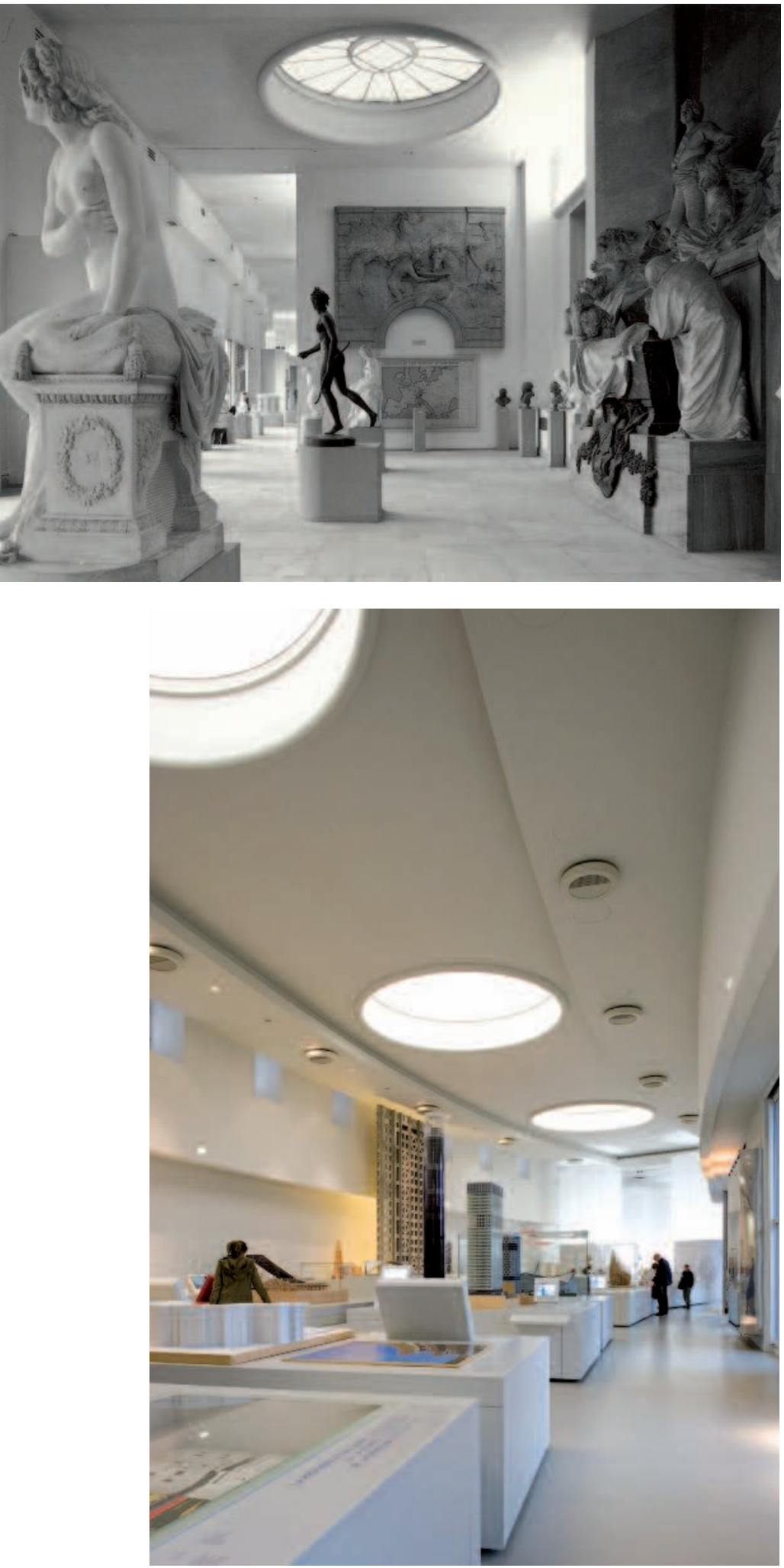

Le niveau supérieur de la galerie Carlu en 1995 et sa transformation en galerie d'Architecture moderne et contemporaine (๑) CAPA/musée des Monuments français ( ) CAPA/musée des Monuments français/N. Borel
Thoronet, les châteaux disparus d'Île-de-France. Pour ceux-ci le découpage séquentiel a été fait avec soin pour permettre une durée de consultation confortable.

Pour la première fois dans un musée, le dispositif de « réalité augmentée » est présenté en salle. La redécouverte de la polychromie des édifices religieux du Moyen Âge fait l'objet depuis quelques années de nombreuses présentations au public, comme la colorisation des sculptures de la façade de la cathédrale d'Amiens par le biais de projections de caches colorés. Le dispositif est installé dans la galerie des Moulages dans la salle «plénitude du Gothique ». Face à la statue du Beau-Dieu de la cathédrale d'Amiens, un écran plat haute définition, encastré dans un mobilier haut, sert de fenêtre dans laquelle la statue reproduite numériquement se pare peu à peu de sa polychromie. Dans un deuxième temps, c'est toute la façade originale qui apparaît remettant la statue dans son contexte sculptural et architectural. Une caméra capte en temps réel la lumière de la galerie. La polychromie se nuance donc selon la lumière extérieure et l'apport d'éclairages artificiels.

Dans la galerie des peintures, des maquettes de contextualisation architecturale sont un élément essentiel du nouveau dispositif muséographique. 24 maquettes de plâtre, à l'échelle $1 / 500^{\circ}$, accompagnent les cartels d'œuvre et indiquent l'emplacement exact de l'œuvre originale dans l'édifice (nef, chapelle, crypte). L'espace occupé par les peintures dans leur édifice peut ainsi être parfaitement appréhendé. Ces maquettes sont réalisées dans la continuité des maquettes en plâtre présentées par la galerie des moulages.

\section{La création d'un nouvel espace : la galerie $d^{\prime}$ 'Architecture moderne et contemporaine}

La galerie de l'Architecture moderne et contemporaine a été créée dans le cadre de la rénovation du musée pour introduire à l'histoire de l'Architecture contemporaine. Ce parcours permanent, consacré à l'Architecture en France de 1851 à nos jours, présente sur $1800 \mathrm{~m}^{2}$ des maquettes créées pour la galerie, des archives audiovisuelles, des photographies et des dessins et plans d'architecte. L'édifice est au centre du discours.

CEuvres et documents sont au service d'une narration qui met en avant l'édifice et traite de la modernité architecturale, montrant davantage des ruptures que des continuités. Les maquettes créées spécialement pour la galerie sont analytiques et didactiques. 
Les extraits de films, en donnant la parole à l'architecte, au commanditaire ou à l'habitant, offrent une mise en contexte historique. Au total, la galerie présente 90 édifices, 100 maquettes, 500 dessins, 90 minutes d'extraits audiovisuels, 140 imprimés, 6 éléments de bâtiment. Les bornes audiovisuelles sont sonorisées, à la différence de celles de la galerie des Moulages. Maquettes, documents, ouvrages sont présentés sur de grandes tables qui mettent les œuvres au niveau du public.

Le pari a été fait de reconstituer, à l'échelle 1 , un appartement en duplex de l'Unité d'habitation de la Cité radieuse de Marseille, selon la conception de son créateur Le Corbusier. Le projet a été réalisé grâce à la participation active de plusieurs lycées professionnels, les lycéens travaillant à la réalisation du projet en accompagnement de la présence des professionnels du bâtiment.

Le public qui redécouvre les salles du musée est tout d'abord pris par la somptuosité du rez-de-chaussée. Les patines des œuvres moulées à l'échelle 1 contribuent à un effet de réel dépaysant et envoûtant. La promenade dans la galerie des Peintures est différente des grandes allées des moulages. Les chapelles se logent dans des circulations complexes. Les motifs surprenants dans leur rendu des chapelles romanes sont à portée d'œil.

La galerie d'Architecture moderne et contemporaine propose un grand nombre de points de vue dans ce qui est un espace relativement petit. Largement ouvert sur les jardins du Trocadéro, la Seine et la Tour Eiffel, le lieu facilite l'approche.

Le musée des Monuments français ne peut se réduire en une approche unique : lieu de présentation de collections patrimoniales, lieu d'enseignement didactique et lieu de dialogue avec le contemporain, ses rôles sont multiples.

\section{Bibliographie}

La Cité de l'Architecture et du Patrimoine, Monumental, Paris : Centre des monuments nationaux, 2007.

Pressouyre, L. (dir.) Le musée des Monuments français. Paris : Éditions Nicolas Chaudun, 2007.

Ory, P. Le Palais de Chaillot. Cité de l'Architecture et du Patrimoine, Aristeas/Acte Sud, 2006.

Collectif. Le musée de Sculpture comparée. Naissance de l'histoire de l'Art moderne, actes du colloque Le musée de Sculpture comparée : l'invention d'un modèle au XIX siècle, 8 et 9 décembre 1999. Paris : Centre des monuments nationaux, Éditions du Patrimoine, 2001. 